\title{
Biodegradación de fenol en aguas tratadas de la industria petrolera para re-uso en cultivos agrícolas
}

\author{
Sergio Pardo-Díaz ${ }^{1}$, Daniel Rojas-Tapias ${ }^{1}$, Fabio Roldan ${ }^{2}$, Pedro Brandão ${ }^{3}$ \& \\ Edgar Almansa-Manrique ${ }^{4}$ \\ 1. Laboratorio de Microbiología de Suelos, Centro de Investigación Tibaitatá. Corpoica, Km 14 vía Mosquera, \\ Colombia; spardo@corpoica.org.co,dfrojas@corpoica.org.co \\ 2. Unidad de Saneamiento y Biotecnología Ambiental (USBA). Pontificia Universidad Javeriana. Carrera $7^{\mathrm{a}} \mathrm{N}^{\circ} 43-82$; \\ fabio.roldan@javeriana.edu.co \\ 3. Laboratorio de Microbiología Ambiental y Aplicada, Departamento de Química, Facultad de Ciencias, Universidad \\ Nacional de Colombia, Ciudad Universitaria - Avenida Carrera 30 º 45-03. Colombia; pfdeb@unal.edu.co \\ 4. Laboratorio de Física de Suelos. Centro de Investigación La Libertad. Corpoica. Km 17 vía Puerto López. Corpoica. \\ Colombia; ealmansa@corpoica.org.co
}

Recibido 27-IV-2016. Corregido 12-XII-2016. Aceptado 16-I-2017.

\begin{abstract}
Biodegradation of phenol in treated water from the oil industry to re-use in agricultural crops. Oil exploitation and the usage of its derivatives have undeniably contributed to the technological advance worldwide. This industrial activity, however, generates several by-products that can threaten environmental sustainability. Seawage, for example, can contain organic pollutants and heavy metals; therefore, its deposition must be preceded by adequate water treatment. In this study, we isolated, characterized, and molecularly identified eight bacteria on the basis of their capability to degrade phenol. First, we determined the rates of bacterial growth and phenol degradation using different concentrations of the aromatic hydrocarbon $(500,800$ and $1200 \mathrm{mg} / \mathrm{L}$ ), and then used sequential statistical designs to select optimal conditions for its degradation. Results showed that all isolated strains were capable of degrading phenol as the sole carbon source; the degradation kinetics and phenol tolerance, however, widely varied among strains. We chose the strain Pseudomonas sp. Sps1 for further studies due to its remarkable tolerance and capability to degrade phenol. By using a Plackett Burman design, followed by a fractionated factorial design in which several carbon, nitrogen, and phosphorus sources were evaluated, and phenol degradation was used as the response variable, we found optimal conditions for phenol degradation by Sps1. Finally, we compared phenol degradation in seawage by the use or not of the optimal conditions established by the statistical methods. Results showed that biostimulation dramatically increased phenol degradation compared with the control. In conclusion, we found that bioaugmentation with the native bacteria Sps1 and a statistically-based biostimulation approach provided an economically and environmentally friendly alternative for the removal of pollutants from oil industry sewage. Rev. Biol. Trop. 65 (2): 685-699. Epub 2017 June 01.
\end{abstract}

Key words: oil extraction, hydrocarbon, native bacteria, nutrients, Colombia.

La explotación de pozos petroleros tiene como consecuencia la producción de grandes cantidades de agua asociada con hidrocarburos. La producción mundial de agua durante la extracción de petróleo se estima de 250 millones de barriles por día en comparación con alrededor de 80 millones de barriles diarios de petróleo, dando como resultado una relación de agua a crudo alrededor de 3:1. El agua de producción a nivel mundial ha aumentado desde hace una década, estimándose que para el 2014 llegue a 200000 barriles por día (Fakhru'l-Razi et al., 2009). En varios países donde la explotación petrolera es de suma importancia económica, la generación de agua es una preocupación debido a los altos costos de su tratamiento y la reincorporación a vertimientos puntuales. Por ejemplo, la descarga de agua de producción en el Mar del Norte (Noruega) alcanzó un volumen anual de cerca de 400 millones de $\mathrm{m}^{3}$ / año 
en 2003 (Durell et al., 2006). En Colombia, en el campo de producción de Castilla la Nueva (Meta), la cantidad de agua para el proceso de extracción de petróleo es de aproximadamente 460000 barriles diarios.

El agua, al estar mezclada con los hidrocarburos, debe pasar por procesos específicos que permitan el máximo aprovechamiento de los productos oleicos que contiene $\mathrm{y}$, por otro lado, debe ser tratada para su adecuado vertimiento al medio ambiente. Arthur, Langhus \& Patel, (2005) proponen cuatro alternativas de disposición final (re-uso) del agua residual tratada de producción para su manejo y disminución del impacto ambiental: 1) re-inyección en los pozos para otras extracciones; 2) descarga en vertientes después de ser tratadas; 3 ) reutilización en los campos de operación como agua para labores diarias; y 4) adecuación para uso agrícola o como agua doméstica.

Los principales compuestos de las aguas residuales de refinería son los fenoles, hidrocarburos aromáticos policíclicos (HAP) y metales pesados. Los fenoles son de gran relevancia en la disposición final de aguas de producción debido a que su presencia en el medio ambiente representa un riesgo importante para la biota acuática, pues es un compuesto muy persistente y letal a bajas concentraciones (5 $25 \mathrm{mg} / \mathrm{L}$ ), lo que representa un riesgo para la salud humana. La ecotoxicidad de los fenoles y compuestos relacionados pueden interferir con el equilibrio del ecosistema y en consecuencia, afectar las vías biogeoquímicas de la materia orgánica y el reciclaje de nutrientes (Contreras, Albertario, Bertola, \& Zaritzky, 2008; CordovaRosa et al., 2009). La toxicidad del fenol está relacionada con la formación de radicales libres y su hidrofobicidad, la cual afecta la solubilidad del fenol en la fracción celular y, por tanto, la posibilidad de interacción del compuesto con las células. Los fenoles, después de la penetración en la célula, sufren una transformación activa, principalmente por oxidasas del citocromo P450. Los procesos de transformación pueden conducir al aumento de la toxicidad por la formación de metabolitos electrófilos que afectan al ADN o las enzimas (Michałowicz \&
Duda, 2007). Existen técnicas fisicoquímicas para la remoción de compuestos fenólicos, sin embargo, estas tienen altos costos y producen otros compuestos tóxicos. Una alternativa es la biorremediación que representa un procedimiento amigable con el medio ambiente. Varios estudios han reportado el aislamiento de microorganismos con la capacidad de degradar fenol a diferentes concentraciones, de las cuales las bacterias se han estudiado ampliamente (Geng Soh, Lim, \& Loke, 2006; Kotresha \& Vidyasagar, 2007; Yang \& Lee, 2007; Dong, Hong, He, Jiang, \& Li, 2008; Cordova-Rosa et al., 2009; Mohite, Jalgaonwala, Pawar, \& Morankar, 2010). Estos estudios han elucidado que los microorganismos se encuentran distribuidos ampliamente en ambientes afectados con fenol, lo que permite pensar en su potencial aplicación para biorremediación de sitios con presencia de este contaminante.

Debido a que los procesos de biorremediación se han constituido en una herramienta importante para descontaminar el medio ambiente, se ha planteado en este trabajo el aislamiento y la caracterización de bacterias con potencial degradador de fenol, y evaluar su capacidad de degradación del compuesto en aguas residuales tratadas de la explotación de petróleo para re-uso en cultivos agrícolas.

\section{MATERIALES Y MÉTODOS}

Muestras ambientales y lugar de muestreo: Las muestras ambientales fueron colectadas (en Noviembre 2013) a partir de biopilas empleadas para remediar lodos aceitosos de fondo de tanque, asociados a procesos de adecuación de aguas de producción de hidrocarburos, localizadas en la estación Castilla la Nueva y Apiay (Meta, Colombia). Se obtuvieron tres muestras compuestas de seis sub-muestras de biopilas con diferentes tiempos de establecimiento: tres biopilas de Castilla con dos años y tres biopilas de Apiay (10 meses, 6 meses y 15 días). Posteriormente, se colectaron muestras de suelos de cultivos de palma y caña de azúcar (Octubre 2014) en el área de Sostenibilidad en Agroenergía ASA-ECOPETROL 
que han sido regados previamente por aguas residuales tratadas de la industria petrolera para re-uso en cultivos agrícolas. Los suelos asociados a palma son suelos con una textura Franco Arcillosa compuestos por arena (67.50\%), limos $(19.16 \%)$ y arcillas $(21.88 \%)$, con $\mathrm{pH}$ 5.31 , un contenido de materia orgánica de 1.71 $\%$, fósforo disponible de $2.30 \mathrm{mg} / \mathrm{kg}$, y nitrógeno orgánico oxidable de $0.71 \%$. Los suelos asociados a caña de azúcar son suelos con una textura Franco Arcillosa arenosa, compuestos por arena $(58.12 \%)$, limos $(20.00 \%)$, arcillas $(21.88 \%)$, con pH 5.16, un contenido de materia orgánica de $1.95 \%$, fósforo disponible de $4.76 \mathrm{mg} / \mathrm{kg}$, y nitrógeno orgánico oxidable de $0.81 \%$. Se obtuvieron muestras compuestas por tres sub-muestras de cada cultivo de $200 \mathrm{~g}$ a $0-20 \mathrm{~cm}$ de profundidad, por triplicado.

\section{Aislamiento de bacterias degradadoras}

de fenol: Para aislar bacterias degradadoras de fenol se usó el medio de cultivo Bushnell-Haas (BH, Bushnell, \& Haas, 1941), suplementado con $500 \mathrm{mg} / \mathrm{L}$ de fenol como única fuente de carbono (Caballero-Mellado, Onofre-Lemus, Estrada-de Los Santos, \& Martínez-Aguilar, 2007; Naresh, Honey, \& Vaishali, 2012). El pH se ajustó a $5.0 \pm 1$ debido a las condiciones de acidez de los suelos de los Llanos Orientales. El fenol se adicionó a partir de una solución madre estéril (filtros $0.22 \mu \mathrm{m}$, Minisart NML) de $100000 \mathrm{mg} / \mathrm{L}$. Las muestras de las biopilas y de los suelos de caña y palma, se procesaron $48 \mathrm{~h}$ después de su recolección; (10 g) de cada muestra se colocaron en $100 \mathrm{~mL}$ de $\mathrm{BH}$ [relación $1: 10(\mathrm{p} / \mathrm{v})$ ] y se incubaron a $28 \pm 2{ }^{\circ} \mathrm{C}$ y $180 \mathrm{rpm}$, realizando tres pases al mismo medio BH fresco cada 21 días (Lin, Reddy, Moorthi, \& Qoma, 2008). Para aislar las cepas promisorias se sembraron diluciones de los cultivos de enriquecimiento en medio $\mathrm{BH}$ sólido (1.8\% agar) fortificado con $500 \mathrm{mg} / \mathrm{L}$ de fenol como única fuente de carbono y energía, se incubó a $28 \pm 2{ }^{\circ} \mathrm{C}$, y se seleccionaron las colonias que crecieron después de cuatro resiembras consecutivas. Las cepas recuperadas se conservaron en $30 \%(\mathrm{v} / \mathrm{v})$ glicerol a $-80{ }^{\circ} \mathrm{C}$.
Degradación de fenol por cepas aisladas: Las cepas degradadoras de fenol se inocularon en caldo de soya tríptica (TSB) a $28 \pm 2{ }^{\circ} \mathrm{C}$ y $120 \mathrm{rpm}$ por $24 \mathrm{~h}$. El cultivo se centrifugó a $5000 \mathrm{rpm}$ por $5 \mathrm{~min}$ y las células fueron lavadas dos veces $[0.85 \%(\mathrm{p} / \mathrm{v}) \mathrm{NaCl}$ estéril]. Para evaluar la degradación de fenol, las células, a una densidad óptica de 1.0 a $600 \mathrm{~nm}\left(\mathrm{OD}_{600}\right)$, fueron inoculadas en medio $\mathrm{BH}$ a una concentración celular del $0.3 \%$ (v / v) en distintas concentraciones de fenol para las cepas aisladas de biopilas $(500 \mathrm{mg} / \mathrm{L}, 800$ mg / L y $1200 \mathrm{mg} /$ L) y de cultivos de caña y palma (500 mg / L y $800 \mathrm{mg} / \mathrm{L}$ ), y se incubaron a $28{ }^{\circ} \mathrm{C} \pm 2$ y $180 \mathrm{rpm}$, hasta agotar la fuente de carbono (Dong et al., 2008). Se evaluó la $\mathrm{OD}_{600}$ de la biomasa y el fenol residual, por el método de Folin-Ciocalteau (Singleton \& Rossi, 1965). Todos los tratamientos se realizaron por triplicado.

Identificación molecular de las cepas: El ADN genómico fue extraído utilizando el kit ZR Fungal / Bacterial DNA MiniPrep (Zymo Research, USA). El gen 16S rRNA se amplificó por PCR con las siguientes condiciones $(50 \mu \mathrm{L})$ : $1 \mathrm{X}$ Taq polimerasa buffer, $1.5 \mathrm{mM} \mathrm{MgCl}, 0.2 \mathrm{mM}$ dNTPs, $0.4 \mu \mathrm{M}$ primer forward 27F (5'- AGAGTTTGATCCTGGCTCAG-3'), $0.4 \mu \mathrm{M}$ primer reverso $1492 \mathrm{R}$ (5'-GGTTACCTTGTTACGACTT-3'), 1.25 U de Taq DNA polimerasa GoTaq ${ }^{\circledR}$ Flexi DNA Polymerase (Promega) y 10-100 ng de ADN genómico. La PCR se realizó en un termociclador (Mastercycler Pro, Eppendorf AG) con el siguiente programa: 2 min a $95^{\circ} \mathrm{C} ; 30$ ciclos de $20 \mathrm{~s}$ a $94{ }^{\circ} \mathrm{C}, 20 \mathrm{~s}$ a $50{ }^{\circ} \mathrm{C}$ y $90 \mathrm{~s}$ a $72{ }^{\circ} \mathrm{C}$; y $10 \mathrm{~min}$ a $72{ }^{\circ} \mathrm{C}$. Los productos de la PCR se secuenciaron comercialmente (Macrogen, Corea). La longitud de las secuencias de $16 \mathrm{~S}$ rRNA fue aproximadamente de 1.5 -kb y fueron cotejadas en GenBank obteniendo los códigos KU291435 a KU291442. Múltiples alineaciones de diferentes secuencias de 16S rRNA de bacterias relacionadas con degradación de fenol de la base de datos GenBank se realizaron con Muscle 7 en la configuración predeterminada. El análisis filogenético se realizó 
mediante el software MEGA7. Las distancias se calcularon utilizando el modelo de Kimura 2-parámetros. Árboles sin enraizar fueron construidos por el método de NeightbourJoining. Se realizó un bootstrap de 1000 veces con el conjunto de datos.

Optimización de condiciones para la biodegradación de fenol: Para optimizar la biodegradación de fenol en aguas de producción de hidrocarburos se utilizaron diseños estadísticos experimentales. El primer diseño usado fue Plackett-Burman como factor de resolución III para evaluar nueve factores nutricionales que influenciaran significativamente en la degradación de fenol. Cada factor se evaluó en dos niveles: -1 para el nivel bajo (ausencia del factor nutricional) y 1 para un nivel alto [presencia en g / L: $0.2 \mathrm{MgSO}_{4} ; 0.034 \mathrm{CaCl}_{2}$; 1.0 fuente de fósforo $\left(\mathrm{KH}_{2} \mathrm{PO}_{4} \mathrm{y} \mathrm{K}_{2} \mathrm{HPO}_{4}\right) ; 1.0$ $\left(\mathrm{NH}_{4}\right)_{2} \mathrm{SO}_{4} ; 0.05 \mathrm{FeCl}_{3} ; 0.5 \mathrm{NaCl} ; 0.1$ micronutrientes $\left(0.1 \mathrm{ZnSO}_{4}, 0.01 \mathrm{NaMo}_{2} \mathrm{O}_{4} ; 0.01\right.$ $\mathrm{CuSO}_{4}$ y $\left.0.01 \mathrm{H}_{3} \mathrm{BO}_{3}\right)$ ]; 0.5 extracto de levadura; y 0.5 glucosa). La variable de respuesta fue la degradación de fenol. Se evaluaron los factores que presentaron un efecto significativo en la degradación de fenol en el diseño de Plackett-Burman, aplicando el Diseño de Factorial Fraccionado (FFD) como factor de resolución IV. Se evaluaron tres niveles $(-1,0$ y 1$)$ para obtener la concentración óptima de las fuentes nutricionales que favorecían la degradación de fenol (Cuadro 1).

CUADRO 1

Factores nutricionales evaluados en el Diseño de Factorial Fraccionado (FFD)

TABLE 1

Nutritional factors evaluated using Fractional factorial design (FFD)

\begin{tabular}{lccc}
\multicolumn{1}{r}{$\begin{array}{c}\text { Fuente Nutricional } \\
(\mathrm{g} / \mathrm{L})\end{array}$} & -1 & 0 & 1 \\
$\mathrm{MgSO}_{4}$ & 0.1 & 0.2 & 0.3 \\
Fuente de Fósforo & 0.5 & 1.0 & 1.5 \\
$\left(\mathrm{NH}_{4}\right)_{2} \mathrm{SO}_{4}$ & 0.5 & 1.0 & 1.5 \\
$\mathrm{NaCl}$ & 0.25 & 0.5 & 0.75 \\
\hline
\end{tabular}

Para evaluar la optimización de las fuentes nutricionales se utilizó agua residual tratada de la explotación de petróleo para re-uso en cultivos agrícolas. Se adicionaron los factores nutricionales $(\mathrm{g} / \mathrm{L})$ que tuvieron un efecto significativo en el FFD y se aplicó fenol a cada cultivo en diferentes concentraciones $(100 \mathrm{mg}$ / L, $250 \mathrm{mg}$ / L y $500 \mathrm{mg}$ / L) cada concentración se evaluó por triplicado. Se plantearon dos tratamientos, uno con la adición de nutrientes y otro con ausencia de los mismos, observando el porcentaje de degradación a las $18 \mathrm{~h}$. Los ensayos se incubaron a $28 \pm 2{ }^{\circ} \mathrm{C}$, sin agitación para simular condiciones de piscina. Como controles se tomó la matriz de cada cultivo sin inocular en presencia y ausencia de los factores nutricionales.

Análisis estadístico: Para el análisis de datos de los diseños experimentales, se utilizó el software estadístico versión 7.0.1 (Stat-Ease Inc., Minneapolis, USA) a un nivel de confianza del $95 \%$.

\section{RESULTADOS}

Aislamiento de cepas asociadas a biopilas: Después de 63 días de enriquecimiento en condiciones de cultivo conteniendo fenol como única fuente de carbono, se recuperaron cuatro cepas de las muestras de biopilas (Sps1, Sps2, $\mathrm{Sc} 3, \mathrm{Sc} 5)$ y cuatro cepas de las muestras de suelo de cultivos agrícolas (ECO1P, ECO2P, Fenol2C y FenolP). La identificación molecular de los aislados y su capacidad de degradación de fenol a diferentes concentraciones se muestran en la cuadro 2 .

La relación filogenética entre las secuencias de 16S rRNA de las cepas aisladas con otras especies de bacterias involucradas en la degradación de fenol se observa en la figura 1 . Se evidenció que la agrupación de la mayoría de las cepas aisladas fueron asociadas al género Pseudomonas: las cepas ECO1P, ECO2P, FenolP y Fenol2C, tuvieron como vecino más cercano las cepas de referencia Pseudomonas plecoglossicia (NR024662); las cepas $\mathrm{Sps} 2$ y Sc3, se asociaron a la cepa de referencia Pseudomonas putida NBRC 14164 


\section{CUADRO 2}

Cepas degradadoras de fenol aisladas de biopilas asociadas a hidrocarburos (BioP) y de suelos de cultivo agrícola regados con aguas de re-uso de la industria petrolera (SAgr)

\section{TABLE 2}

Phenol-Degrading strains isolated from hydrocarbon associated biopiles (BioP) and agricultural crop soils irrigated with re-use water from oil production industry (SAgr)

\begin{tabular}{lllccc}
\multicolumn{1}{c}{ Origen } & \multicolumn{1}{c}{ Cepa } & \multicolumn{1}{c}{ Identificación molecular } & \% Similitud* & Código GenBank & Degradación de fenol $^{\#}$ \\
BioP & Sps1 & Pseudomonas sp. & 99 & KU291435 & +++ \\
& Sps2 & Pseudomonas putida & 100 & KU291437 & ++ \\
& Sc3 & Pseudomonas putida & 100 & KU291438 & ++ \\
& Sc5 & Achromobacter sp. & 99 & KU291436 & ++ \\
SAgr & ECO1P & Pseudomonas sp. & 100 & KU291439 & + \\
& ECO2P & Pseudomonas sp. & 100 & KU291440 & + \\
& Fenol2C & Pseudomonas sp. & 100 & KU291441 & + \\
\hline
\end{tabular}

* Porcentaje de similitud con la secuencia más cercana en GenBank. Similarity percentage with the most related sequence in GenBank database. (http://www.ncbi.nlm.nih.gov/genbank/)

\# Degradación de fenol a $1200 \mathrm{mg} / \mathrm{L}(+++), 800 \mathrm{mg} / \mathrm{L}(++)$ o $500 \mathrm{mg} / \mathrm{L}(+) .1200 \mathrm{mg} / \mathrm{L}(+++), 800 \mathrm{mg} / \mathrm{L}(++)$ or $500 \mathrm{mg} / \mathrm{L}(+)$ phenol-degradation.

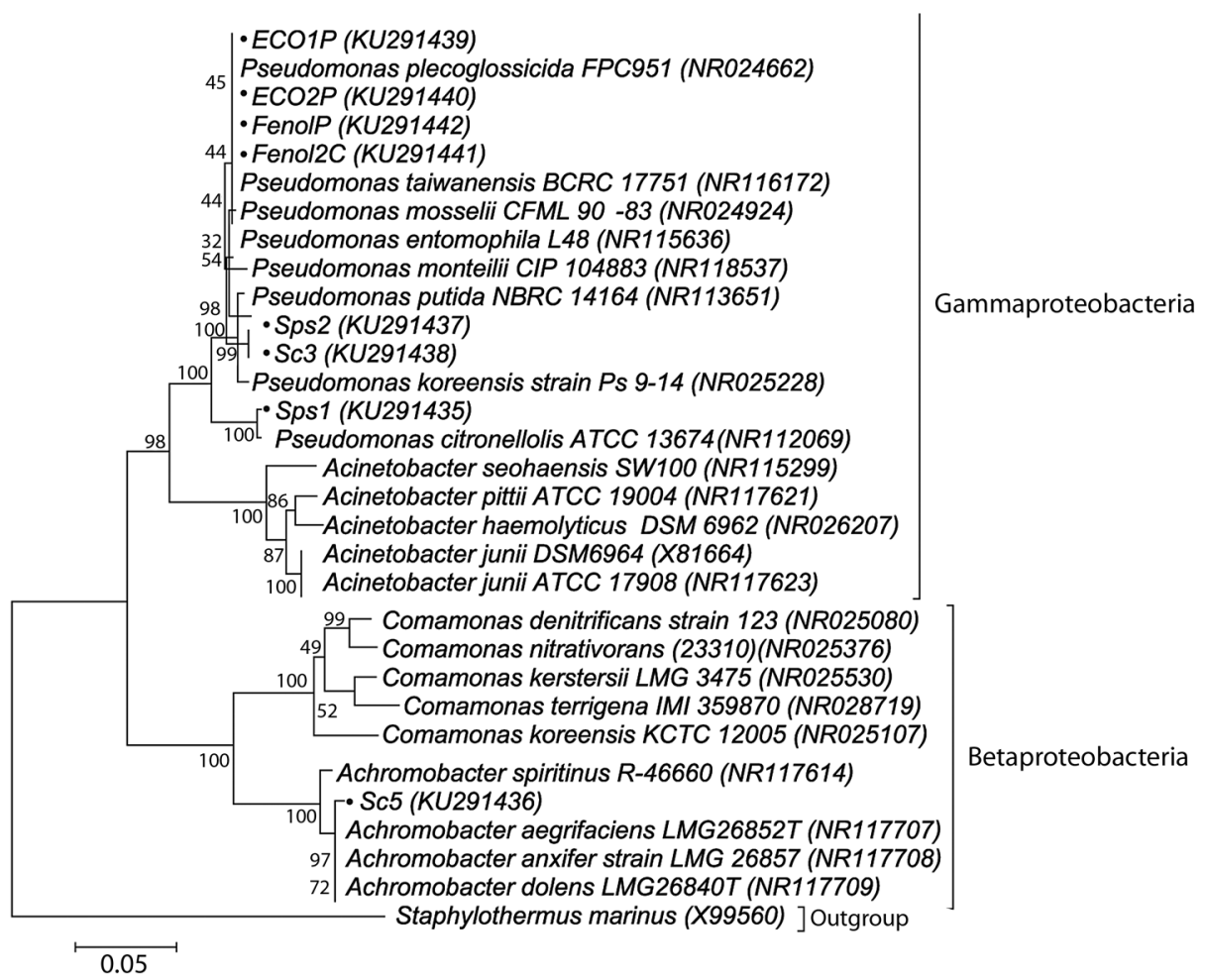

Fig. 1. Análisis filogenético basado en las secuencias de $16 \mathrm{~S}$ rDNA de las cepas degradadoras de fenol y especies relacionadas. El método de Neightbour-Joining con el modelo de Kimura 2-parameter en MEGA7 fue empleado para la construcción del árbol y los valores de bootstrap obtenido con 1000 repeticiones se indican como porcentajes en los nodos. Fig. 1. Phylogenetic analysis based in $16 \mathrm{~S}$ rDNA sequences of the phenol-degrading strains and related species. The Neightbour-joining method with Kimura 2-parameter model in MEGA7 were used for tree construction and bootstrap values obtained with 1000 replications are indicated as percentage at the nodes. 
(NR113651); y la cepa Sps1, se asoció a la cepa de referencia Pseudomonas citronellolis ATCC 13674 (NR112069). La cepa Sc5 fue asociada a diferentes especies del género Achromobacter: A. aegrifaciens LMG 26852T (NR117707); A. anxifer LMG 26857 (NR117708); y A. dolens LMG 26840T (NR117709).

Degradación de fenol por cultivos puros: Las cinéticas de degradación a distintas concentraciones de fenol en el tiempo por una selección de cepas recuperadas de biopilas, junto con el crecimiento observado para cada caso en las condiciones de cultivo utilizadas, se muestran en la figura 2. Se observó que las cepas Sps1 y Sc3 removieron por completo el fenol a $500 \mathrm{mg} / \mathrm{L}$ del medio de cultivo a las $24 \mathrm{~h}$ y $28 \mathrm{~h}$, respectivamente (Fig. 2A). Estas cepas, a pesar de requerir un periodo de adaptación más largo a $800 \mathrm{mg} / \mathrm{L}$ de fenol, degradaron el compuesto por completo a las 48 h (Fig. 2B). La cepa Sps1 fue la única que creció a $1200 \mathrm{mg} / \mathrm{L}$ de fenol, removiendo
A

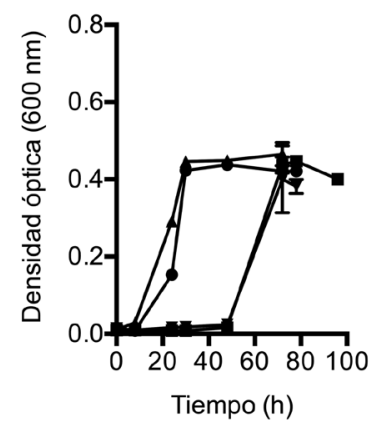

B

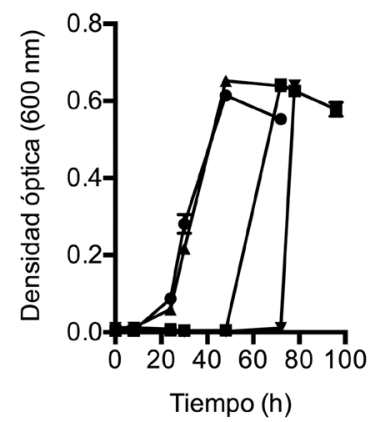

C

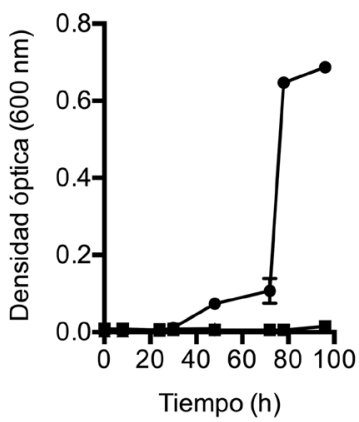

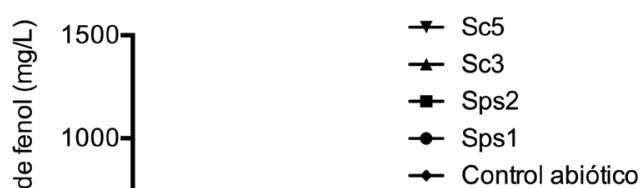

500

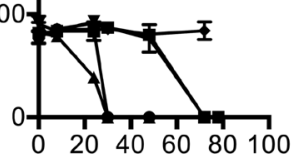

Tiempo (h)
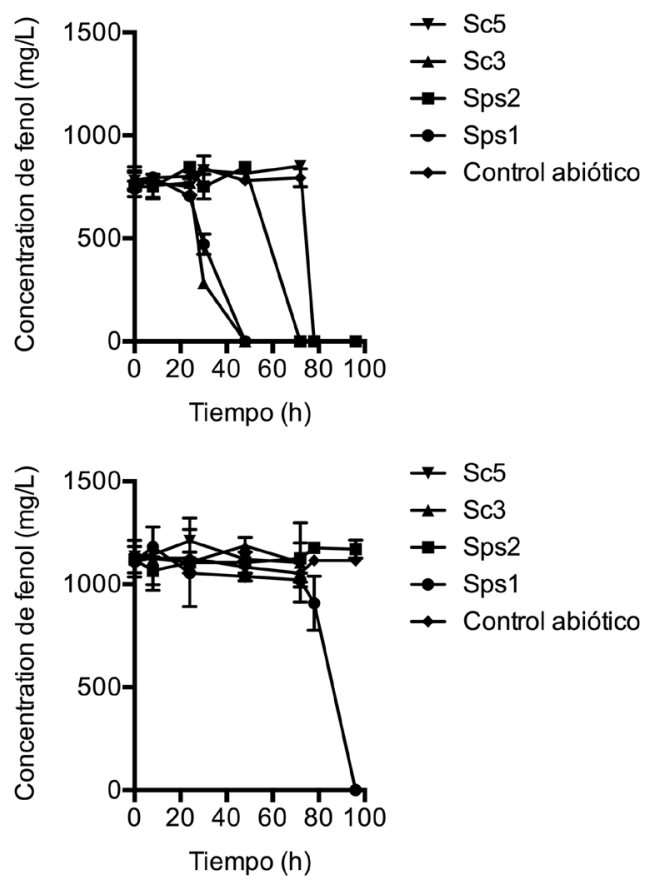

Fig. 2. Cinéticas de crecimiento (izquierda) y remoción de fenol (derecha) de las cepas recuperadas de biopilas a diferentes concentraciones de fenol: A) $500 \mathrm{mg} / \mathrm{L}$, B) $800 \mathrm{mg} / \mathrm{L} \mathrm{y} \mathrm{C)} 1200 \mathrm{mg} / \mathrm{L}$. Las barras de error corresponden al desvío estándar obtenido de análisis por triplicado. Control: control abiótico de fenol.

Fig. 2. Growth kinetics (left) and phenol removal (right) of strains isolated from biopiles at different phenol concentrations, a) $500 \mathrm{mg} / \mathrm{L}$, b) $800 \mathrm{mg} / \mathrm{L} \mathrm{y} \mathrm{c)} 1200 \mathrm{mg} / \mathrm{L}$. Bars indicate standard deviation. Control: phenol abiotic control. 
totalmente el compuesto a las $96 \mathrm{~h}$; para el resto de las cepas esta concentración inhibió su crecimiento (Fig. 2C).

Las cinéticas de degradación de las cepas recuperadas de suelos de interés agrícola (caña y palma) se observan en la figura 3. La cepa ECO1P presentó un menor tiempo de adaptación en comparación con las demás, logrando remover la totalidad de $500 \mathrm{mg} / \mathrm{L}$ fenol en 28 h. Para las demás cepas, la remoción total de fenol se evidenció después de 46 h. A 800 y 1 $200 \mathrm{mg} / \mathrm{L}$ de fenol se inhibió el crecimiento microbiano y no se evidenció remoción de fenol en el tiempo (no mostrado).

Se observó que la biomasa celular aumentó cuando se incrementó la concentración de fenol de 500 a 800 mg / L (Fig. 2A y Fig. 2B). En todos los controles abióticos la concentración de fenol se mantuvo constante, evidenciando que su degradación en los ensayos bióticos fue realizada por los microorganismos. Con el incremento de la concentración de fenol se observó de forma general que la fase de adaptación de las cepas al medio de cultivo aumentó. Sin embargo, las cepas Sps1, Sc3 y ECO1P, presentaron una fase de adaptación $(<10 \mathrm{~h})$ inferior a las demás (Fig. 2 y Fig. 3).

Optimización de condiciones para la biodegradación de fenol: Diseños estadísticos fueron empleados para identificar factores nutricionales limitantes en aguas de re-uso de explotación de hidrocarburos. Se seleccionó la cepa Sps1 para desarrollar los diseños porque fue la que mostró mejor capacidad de degradación de fenol. Para ello se evaluaron nueve factores nutricionales en el diseño de Placket-Burman y su variable de respuesta fue la degradación de fenol (Fig. 4).

Las estimaciones de los parámetros de los componentes se muestran en el cuadro 3. La glucosa, los micronutrientes y el $\mathrm{CaCl}_{2}$, presentaron un nivel de significancia negativa que afectó la variable de respuesta por lo cual se omitieron para continuar con el diseño de la optimización. Las fuentes nutricionales que mostraron un nivel de significancia positivo (Extracto de levadura, $\left(\mathrm{NH}_{4}\right)_{2} \mathrm{SO}_{4}$, fuentes de fósforo $\left(\mathrm{KH}_{2} \mathrm{PO}_{4} \mathrm{y}\right.$ $\left.\mathrm{K}_{2} \mathrm{HPO}_{4}\right), \mathrm{MgSO}_{4}$ y $\mathrm{NaCl}$ ) se usaron para el siguiente diseño. $\mathrm{El} \mathrm{FeCl}_{3}$ presentó un efecto positivo pero no significativo para la variable de respuesta por lo cual se omitió del ensayo.

Para continuar con la evaluación de los factores nutricionales que presentaron una respuesta positiva en el diseño de Plackett-Burman se utilizó el Diseño de Factorial Fraccionado (FFD). Este diseño es de resolución IV lo que significa que los efectos principales no se confunden, ya sea con otros efectos principales o interacciones de dos factores (Ren et al., 2008). Los factores nutricionales evaluados en el diseño FFD se muestran en la figura 5. Las estimaciones
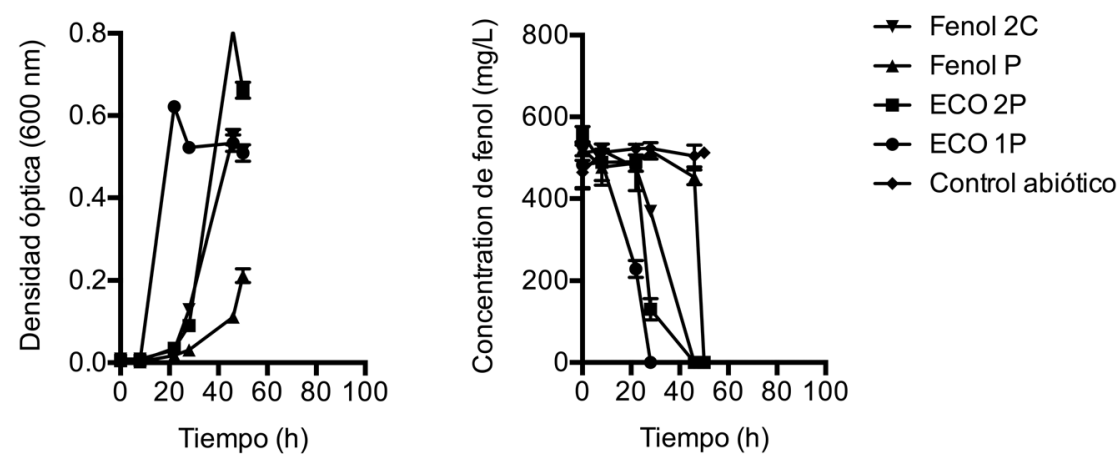

Fig. 3. Cinéticas de crecimiento (izquierda) y remoción de $500 \mathrm{mg} / \mathrm{L}$ fenol (derecha) de las cepas aisladas de las muestras de suelo de cultivos agrícolas. Las barras de error corresponden al desvío estándar obtenido de análisis por triplicado. Control: control abiótico de fenol.

Fig. 3. Growth kinetics (left) and $500 \mathrm{mg} / \mathrm{L}$ phenol removal (right) of strains isolated from agricultural crop soils. Bars indicate standard deviation. Control: phenol abiotic control. 


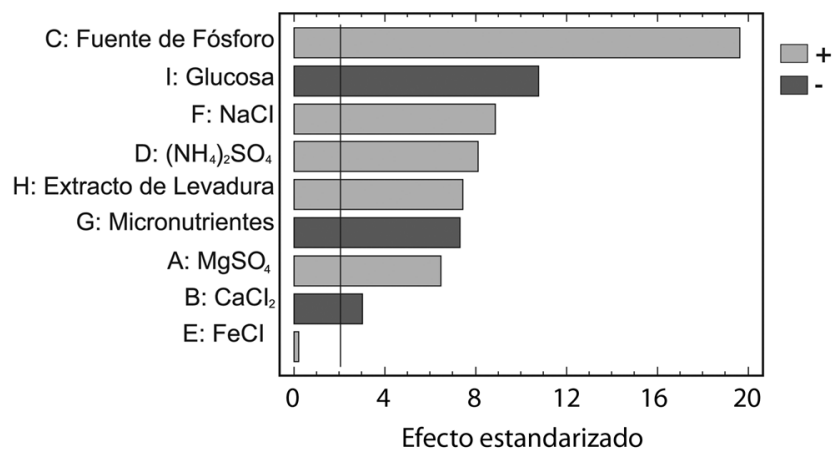

Fig. 4. Pareto del diseño de Plackett-Burman para identificar factores nutricionales limitantes en la degradación de fenol en aguas de re-uso de explotación de hidrocarburos. R2 =97.11\%; Adj-R2 =96.11\%; Nivel de confianza $=95 \%$.

Fig. 4. Pareto chart of Plackett-Burman design to identify limiting nutritional factors of phenol-degradation in re-use water from oil production industry. R2 $=97.11 \%$; Adj-R2 $=96.11 \%$; Confidence level $=95 \%$.

\section{CUADRO 3}

Estimaciones y resultados del diseño Plackett-Burman para identificar las mejores condiciones para la degradación de fenol en aguas de re-uso de explotación de hidrocarburos

TABLE 3

Estimations and results of Plackett-Burman design to identify optimal conditions for phenol-degradation in re-use water from oil production industry

\begin{tabular}{cccccccccccc} 
Tratamiento & A & B & C & D & E & F & G & H & I & \multicolumn{2}{c}{ Degradación de Fenol (\%) } \\
1 & -1 & -1 & -1 & -1 & -1 & -1 & -1 & -1 & -1 & 7.00 & 6.93 \\
2 & 1 & 1 & -1 & 1 & -1 & -1 & -1 & 1 & 1 & 30.80 & 24.76 \\
3 & 1 & -1 & 1 & 1 & -1 & 1 & -1 & -1 & -1 & 100.00 & 100.44 \\
4 & -1 & 1 & 1 & 1 & -1 & 1 & 1 & -1 & 1 & 44.80 & 40.80 \\
5 & 1 & -1 & -1 & -1 & 1 & 1 & 1 & -1 & 1 & 8.80 & 1.29 \\
6 & -1 & 1 & 1 & -1 & 1 & -1 & -1 & -1 & 1 & 18.20 & 20.24 \\
7 & 1 & 1 & 1 & -1 & 1 & 1 & -1 & 1 & -1 & 92.40 & 92.93 \\
8 & -1 & 1 & -1 & -1 & -1 & 1 & 1 & 1 & -1 & 21.00 & 19.98 \\
9 & -1 & -1 & 1 & 1 & 1 & -1 & 1 & 1 & -1 & 76.20 & 67.89 \\
10 & 1 & -1 & 1 & -1 & -1 & -1 & 1 & 1 & 1 & 31.40 & 40.56 \\
12 & -1 & -1 & -1 & 1 & 1 & 1 & -1 & 1 & 1 & 28.40 & 36.82 \\
\hline
\end{tabular}

Ver figura 4 para identificar las fuentes nutricionales evaluadas (A-I).

Figure 4 shows the nutritional sources evaluated (A-I).

de los parámetros de los componentes para el diseño FFD se muestran en el cuadro 4.

Los factores que presentaron un nivel de significancia positivo, reflejados en el porcentaje de degradación de fenol, fueron las fuentes de fosforo $\left(\mathrm{KH}_{2} \mathrm{PO}_{4}\right.$ y $\left.\mathrm{K}_{2} \mathrm{HPO}_{4}\right)$ y la fuente de nitrógeno
$\left(\left(\mathrm{NH}_{4}\right)_{2} \mathrm{SO}_{4}\right)$. Se observó que los factores tuvieron el efecto positivo más alto en la variable de respuesta, cuando la concentración ( $\mathrm{g} / \mathrm{L}$ ) fue la más alta probada en el ensayo. Los demás factores evaluados no fueron significativos en la degradación de fenol. 


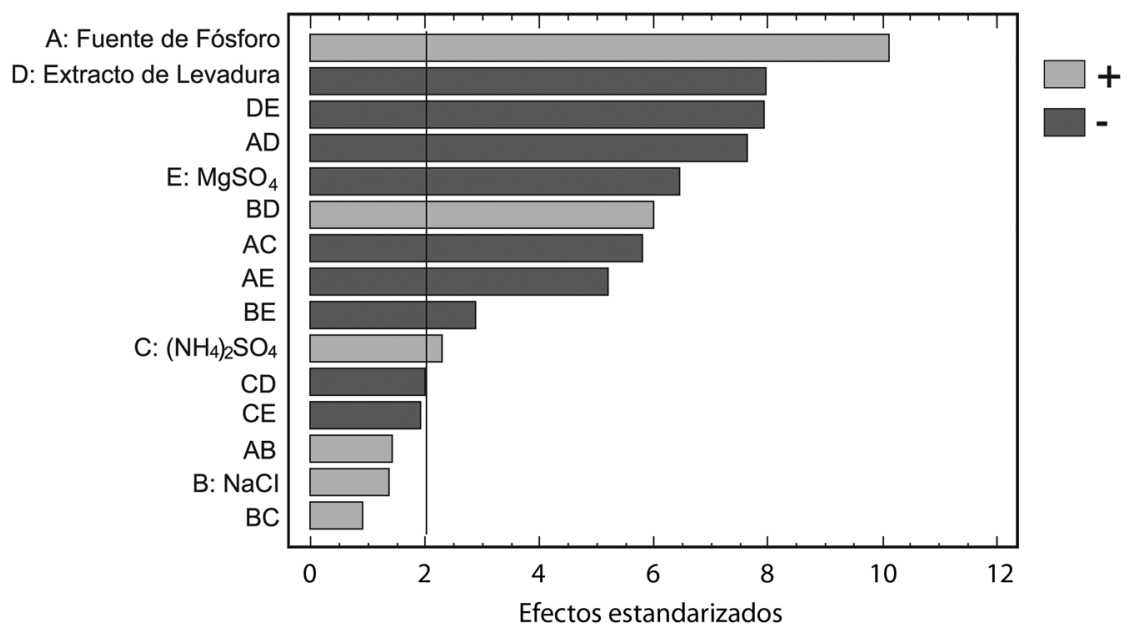

Fig. 5. Pareto del diseño FFD para identificar factores nutricionales limitantes en la degradación de fenol en aguas de re-uso de explotación de hidrocarburos. R2 = $92.85 \%$; Adj-R2 = 89.79\%; Nivel de confianza $=95 \%$.

Fig. 5. FFD pareto design to identify limiting nutritional factors of phenol degradation in re-use water from oil production industry. R2 = $92.85 \%$; Adj-R2 = 89.79 \%; Confidence level $=95 \%$.

\section{CUADRO 4}

Estimaciones y resultados del diseño FFD y sus niveles para identificar las mejores condiciones para la degradación de fenol en aguas de re-uso de explotación de hidrocarburos

\section{TABLE 4}

Estimations and results of FFD design with factor levels to identify optimal conditions for phenol-degradation in re-use water from oil production industry

\begin{tabular}{cccccccc} 
Tratamiento & A & B & C & D & E & \multicolumn{2}{c}{ Degradación de fenol (\%) } \\
\hline 1 & -1 & 1 & -1 & 1 & 1 & 72.93 & Observada \\
2 & -1 & -1 & -1 & -1 & 1 & 84.21 & 81.16 \\
3 & -1 & 1 & -1 & -1 & -1 & 58.90 & 62.23 \\
4 & -1 & -1 & -1 & 1 & -1 & 73.06 & 71.58 \\
5 & 1 & -1 & 1 & 1 & -1 & 79.36 & 80.81 \\
6 & 1 & -1 & -1 & -1 & -1 & 101.65 & 100.48 \\
7 & 1 & 1 & 1 & 1 & 1 & 67.82 & 66.47 \\
8 & 0 & 0 & 0 & 0 & 0 & 69.13 & 82.06 \\
9 & -1 & 1 & 1 & 1 & -1 & 92.87 & 92.09 \\
10 & -1 & -1 & 1 & 1 & 1 & 70.57 & 70.27 \\
11 & 1 & 1 & -1 & -1 & 1 & 94.96 & 93.22 \\
12 & 1 & 1 & 1 & -1 & -1 & 101.91 & 80.61 \\
13 & -1 & 1 & 1 & -1 & 1 & 79.10 & 96.28 \\
14 & 1 & -1 & 1 & -1 & 1 & 97.85 & 99.34 \\
15 & 1 & 1 & -1 & 1 & -1 & 100.34 & 68.26 \\
16 & 1 & -1 & -1 & 1 & 1 & 70.31 & 80.72 \\
\hline
\end{tabular}

Ver cuadro 1 para identificar las fuentes nutricionales evaluadas (A-E).

Table 1 show nutritional sources evaluated (A-E). 
Efecto de la optimización de degradación de fenol en aguas residuales tratadas de la explotación de petróleo: Para el establecimiento de las condiciones nutricionales que mejoran la degradación de fenol se consideraron los factores que presentaron significancia en el diseño FFD. Se optimizaron las condiciones utilizando $1.5 \mathrm{~g} / \mathrm{L} \mathrm{KH}_{2} \mathrm{PO}_{4}, 1.5 \mathrm{~g} / \mathrm{L}$ $\mathrm{K}_{2} \mathrm{HPO}_{4}$ y $1.5 \mathrm{~g} / \mathrm{L}\left(\mathrm{NH}_{4}\right)_{2} \mathrm{SO}_{4}$. Estas condiciones nutricionales se evaluaron en aguas tratadas provenientes de re-uso de la industria petrolera.
En las ocho cepas evaluadas se evidenció la capacidad de degradación de fenol en presencia de los nutrientes bajo concentraciones de 100 $\mathrm{mg} / \mathrm{L}$ y $250 \mathrm{mg} / \mathrm{L}$ en un rango del $96.93 \%$ al $100 \%$ a las $18 \mathrm{~h}$, exceptuando la cepa Sc5 que presentó un porcentaje bajo de degradación en todas las concentraciones evaluadas (Cuadro 5). La cepa Sc3 presentó mayor degradación de fenol (46.09\%) en la concentración más alta evaluada (500 mg / L). El grupo de cepas asociados a los cultivos agrícolas (caña

CUADRO 5

Porcentajes de degradación de fenol en aguas tratadas provenientes de re-uso de la industria petrolera por las cepas aisladas, utilizando los factores nutricionales optimizados por diseño FFD

TABLE 5

Phenol-degradation percentage in re-use water from oil production industry with isolated strain, using nutritional factors optimized by FFD design

\begin{tabular}{|c|c|c|c|}
\hline Cepa & Fenol (mg / L) & \% Degradación CN & \% Degradación SN \\
\hline \multirow[t]{3}{*}{ Sps1 } & 100 & $100.00 \pm 0.655$ & $0.00 \pm 0.013$ \\
\hline & 250 & $100.00 \pm 0.400$ & $0.00 \pm 0.033$ \\
\hline & 500 & $33.24 \pm 2.760$ & $0.00 \pm 0.042$ \\
\hline \multirow[t]{3}{*}{ Sps2 } & 100 & $100.00 \pm 0.005$ & $0.00 \pm 0.026$ \\
\hline & 250 & $96.93 \pm 3.67$ & $0.00 \pm 0.085$ \\
\hline & 500 & $25.00 \pm 1.44$ & $0.00 \pm 0.032$ \\
\hline \multirow[t]{3}{*}{$\mathrm{Sc} 3$} & 100 & $100.00 \pm 0.65$ & $0.00 \pm 0.064$ \\
\hline & 250 & $100.00 \pm 0.302$ & $0.00 \pm 0.087$ \\
\hline & 500 & $46.09 \pm 1.491$ & $0.00 \pm 0.128$ \\
\hline \multirow[t]{3}{*}{ Sc5 } & 100 & $11.65 \pm 1.000$ & $0.00 \pm 0.075$ \\
\hline & 250 & $4.42 \pm 0.262$ & $0.00 \pm 0.127$ \\
\hline & 500 & $2.59 \pm 0.200$ & $0.00 \pm 0.162$ \\
\hline \multirow[t]{3}{*}{ ECO1P } & 100 & $100.00 \pm 0.007$ & $46.16 \pm 0.008$ \\
\hline & 250 & $96.40 \pm 0.021$ & $12.40 \pm 0.039$ \\
\hline & 500 & $40.20 \pm 0.398$ & $7.30 \pm 0.193$ \\
\hline \multirow[t]{3}{*}{$\mathrm{ECO} 2 \mathrm{P}$} & 100 & $100.00 \pm 0.005$ & $34.41 \pm 0.020$ \\
\hline & 250 & $100.00 \pm 0.071$ & $12.90 \pm 0.028$ \\
\hline & 500 & $31.10 \pm 0.138$ & $5.90 \pm 0.058$ \\
\hline \multirow[t]{3}{*}{ Fenol2C } & 100 & $100.00 \pm 0.006$ & $28.27 \pm 0.090$ \\
\hline & 250 & $100.00 \pm 0.011$ & $12.90 \pm 0.019$ \\
\hline & 500 & $6.30 \pm 0.178$ & $0.00 \pm 0.063$ \\
\hline \multirow[t]{3}{*}{ FenolP } & 100 & $100.00 \pm 0.010$ & $42.40 \pm 0.016$ \\
\hline & 250 & $100.00 \pm 0.014$ & $5.20 \pm 0.084$ \\
\hline & 500 & $21.70 \pm 0.369$ & $0.00 \pm 0.184$ \\
\hline \multirow[t]{3}{*}{ Control } & 100 & $19.36 \pm 0.020$ & $0.00 \pm 0.030$ \\
\hline & 250 & $0.00 \pm 0.050$ & $0.00 \pm 0.030$ \\
\hline & 500 & $3.70 \pm 0.257$ & $0.00 \pm 0.125$ \\
\hline
\end{tabular}

$\mathrm{CN}$ : Con nutrientes; SN: Sin Nutrientes.

$\mathrm{CN}$ : With Nutrients; SN: Without nutrients. 
y palma) presentaron una menor degradación a través del tiempo en la concentración más alta evaluada. Los controles sin inocular presentaron un porcentaje nulo o bajo de degradación. En los tratamientos sin nutrientes se evidenció una remoción de fenol no mayor del $46 \%$ en la concentración de $100 \mathrm{mg} / \mathrm{L}$. Para todas las cepas el \% degradación en ausencia de los nutrientes $(\mathrm{SN})$ fue nulo o siempre inferior al tratamiento con nutrientes $(\mathrm{CN})$.

\section{DISCUSIÓN}

Debido a que después de realizar procesos químicos y mecánicos previos no se remueven completamente los fenoles contenidos en aguas tratadas de la industria petrolera, la búsqueda y utilización de nuevas alternativas como microorganismos con la capacidad de degradar fenol para la descontaminación de las mismas, se hace una herramienta importante para la remoción de este contaminante que en contacto con el ambiente representa un riesgo para comunidades acuáticas y terrestres. La utilización de microorganismos degradadores de fenol está descrita ampliamente en varios estudios que muestran resultados relevantes frente a este contaminante (Geng et al., 2006; Kotresha \& Vidyasagar, 2007; Yang \& Lee, 2007; Dong et al., 2008; Mohite et al., 2010). Teniendo en cuenta que el fenol es persistente en aguas de re-uso provenientes de explotación de hidrocarburos, la utilización de microorganismos es una alternativa potencial que puede ser aplicada para descontaminar este recurso, que tiende a ser escaso y por lo tanto costoso con el tiempo. Las cepas degradadoras de fenol recuperadas en este estudio pertenecen a géneros taxonómicos anteriormente reportados como bacterias degradadoras de fenoles y de otros hidrocarburos (Bandyopadhyay, Das, \& Maiti, 1998; Quan, Shi, Liu, Wang, \& Qian, 2004; Banerjee \& Ghoshal, 2010; Sridevi, Lakshmi, \& Swamy, 2011). Particularmente, se encuentra a Pseudomonas sp. como el género más reportado. Estudios recientes basados en bacterias cultivables sugiere que en suelos afectados con hidrocarburos predominan bacterias
Gram-negativas (Jain et al., 2005), lo que está de acuerdo con este estudio donde todas las cepas recuperadas fueron Gram-negativas.

En el análisis filogenético basado en las secuencias del gen 16S rRNA evidenció la presencia de distintas especies del género Pseudomonas. Los microorganismos pertenecientes al grupo Pseudomonales son conocidos por su capacidad de degradación aeróbica de una serie de hidrocarburos, compuestos aromáticos, y sus derivados, entre los cuales hay compuestos naturales y productos finales o intermedios procedentes de actividades industriales. Por otro lado, el género Achromobacter sp., al cual pertenece la otra cepa identificada en este trabajo, ha sido reportado en procesos de degradación del 2,4-diclorofenol en cultivos puros, en condiciones de biorreactor (Airlift), la cual ha originado una tasa de remoción de $1.24 \mathrm{mg}$ / h en los dos primeros días (Quan et al., 2004). Nair, Jayachandran y Shashidhar (2008), reportan este género dentro de un gran grupo de microorganismos como biodegradador de fenol. Ho et al. (2012), inocularon Achromobacter xylosidans F3B en Arabidopsis thaliana para disminuir la fitotoxicidad del fenol ejercida sobre la planta, observando una disminución de la concentración de fenol evaluada.

Las cepas aisladas en este estudio fueran evaluadas en tres concentraciones diferentes de fenol (500 mg / L, $800 \mathrm{mg} / \mathrm{L}$ y $1200 \mathrm{mg}$ / L) observándose distintos tiempos de degradación, adaptación al fenol y producción de biomasa. Se observó que el aumento de biomasa está asociado a la disminución de fenol en el medio, indicando que el fenol es utilizado como fuente de carbono. La habilidad de los microorganismos para incorporar fenol para producción de biomasa, está relacionada con la expresión de enzimas (hidroxilasas) asociadas a la degradación del compuesto, lo cual forma catecol que es transformado por medio de las enzimas $\mathrm{C} 230$ o $\mathrm{C} 120$ a piruvato, para ser utilizado en el metabolismo celular (Dong et al., 2008). Entre las cepas aisladas, la cepa Sps1 presentó la mayor tolerancia al fenol, pues lo degradó totalmente en $96 \mathrm{~h}$ a la concentración 
más elevada evaluada (1200 mg / L); esta concentración inhibió el crecimiento y la degradación de las demás cepas recuperadas de biopilas. Para las cepas aisladas de cultivos agrícolas la concentración de 800 y $1200 \mathrm{mg} /$ L presentó inhibición de crecimiento y degradación. Es posible que el fenol ejerza un efecto tóxico a nivel de membrana, lo que puede ser soportado por observaciones de los cambios en función de la membrana y la influencia de la relación proteína-lípido de la membrana (Sikkema, Bont, \& Poolman, 1995). Además, la acción inhibidora en la mayoría de los casos está directamente relacionada con el comportamiento de partición de los compuestos lipófilos en $n$-octanol / agua, lo que sugiere que la membrana citoplásmica es el sitio primario de acción tóxica (Sikkema et al., 1995). Dong et al. (2008) evaluaron la capacidad de degradación de cepas aisladas de suelos sin cultivar en distintas concentraciones de fenol (1-10 mM), y observaron que el aumento de la concentración inicial del compuesto en el medio de cultivo aumentaba proporcionalmente el período de adaptación de las cepas comparado con las que crecían en concentraciones más bajas, esto se debe al efecto inhibitorio del compuesto en concentraciones altas. Además, el aumento máximo de la biomasa fue observado en las altas concentraciones de fenol debido a su mayor disponibilidad como única fuente de carbono y energía. De forma similar, Yang y Lee (2007) evaluaron la remoción de 100 a $600 \mathrm{mg} / \mathrm{L}$ de fenol (1 mM a $6 \mathrm{mM}$, respectivamente) utilizando cultivos puros de Pseudomonas resinovorans cepa P-1 y Brevibacillus sp. cepa P-6, aisladas de suelos contaminados con pentaclorofenol. Los autores observaron un aumento en la fase de adaptación y encontraron que en las concentraciones más altas, las cepas presentaban inhibición de crecimiento y capacidad de remoción de fenol. Los resultados obtenidos en este estudio sugieren que el tiempo de adaptación de las bacterias y la concentración del fenol están relacionados con el tiempo de degradación de fenol por parte de los microorganismos, lo que indica que las cepas con un menor tiempo de adaptación podrán remover exitosamente el compuesto de forma más rápida. Masque, Nolla, Bordons, Tarragona y Barcelona (1987) observaron que la adaptación a fenol (1000 mg / L) por una Pseudomonas sp. disminuía a medida que las células eran incubadas y pre-adaptadas a concentraciones más bajas de fenol, obteniendo así una degradación en menor tiempo. Estos resultados son concordantes con los obtenidos en el presente trabajo, donde se observó que el aumento de biomasa correspondió a la disminución de fenol, indicando que las cepas aisladas poseen relevancia en la degradación de fenol.

Con los resultados de las cinéticas de degradación de fenol por las cepas aisladas, se buscaron alternativas para mejorar la remoción de fenol en aguas residuales tratadas de la explotación de petróleo para re-uso en cultivos agrícolas. Esto se realizó una vez que en estudios previos (datos no mostrados) se observó que estas aguas tenían un contenido bajo de nutrientes implicados en el metabolismo microbiano y degradación de fenol. El diseño Plackett-Burman es una forma eficaz de identificar los factores importantes entre un gran número de variables (Stanbury, Whitaker, \& Hall, 1986). Este diseño es de resolución III, lo que significa que los efectos principales no se confunden con otros efectos principales, pero son confundidos con una o más interacciones en dos sentidos, que deben ser asumidas como cero, para que los efectos principales sean significativos. Posteriormente, se continuó con el análisis de las variables que presentaron un efecto positivo sobre la variable de respuesta (degradación de fenol), y para ello se empleó el FFD. Este diseño es de resolución IV, lo que significa que los efectos principales no se confunden, ya sea con otros efectos principales o interacciones de dos factores, aunque las interacciones de dos factores pueden ser confundidas con otros dos factores de interacción (Ren et al., 2008). Se observó con el diseño de Plackett-Burman que la glucosa, los micronutrientes y el $\mathrm{CaCl}_{2}$ presentaron un nivel de significancia negativa que afectó la degradación del fenol. La presencia de glucosa puede inhibir la síntesis de enzimas 
responsable por la utilización de fenol por las células, debido a que la glucosa afecta el sistema de transporte del fenol a través de la membrana por la afinidad de la membrana a la glucosa (Annadurai et al., 2008). Se tomaron las fuentes que mostraron una significancia positiva para la degradación de fenol y se diseñó el FFD, el cual indicó que los factores nutricionales significativos para la degradación de fenol fueron las fuentes de fósforo $\left(\mathrm{KH}_{2} \mathrm{PO}_{4} \mathrm{y}\right.$ $\left.\mathrm{K}_{2} \mathrm{HPO}_{4}\right)$ y de nitrógeno $\left(\left(\mathrm{NH}_{4}\right)_{2} \mathrm{SO}_{4}\right)$, mientras que el extracto de levadura y el $\mathrm{NaCl}$ presentaron un nivel negativo. Las fuentes de fosforo son importantes para el metabolismo microbiano porque están involucradas en la síntesis de ácidos nucleicos y de fosfolípidos relacionados con la membrana citoplasmática de las células, la cual forma una matriz en la que las enzimas y el transporte de proteínas están integrados, estructuras que permiten la captación selectiva de solutos y su excreción. Además de su rol en el transporte de solutos, la membrana citoplasmática juega un papel crucial en el mantenimiento del estado de energía de la célula, la regulación del medio ambiente intracelular, la presión de turgencia, transducción de señales, y otros procesos de transducción de energía (Sikkema et al., 1995). En el presente estudio, se observó que la fuente de nitrógeno $\left(\left(\mathrm{NH}_{4}\right)_{2} \mathrm{SO}_{4}\right)$ está asociada con el máximo de respuesta de degradación de fenol. El nitrógeno juega un papel importante en la determinación de los tipos y el alcance de la degradación química (Jung \& Neal, 1991). Annadurai et al. (2008) reportaron que el sulfato de amonio, como suplemento en medio mineral, disminuyó significativamente la toxicidad del fenol e incrementó la densidad celular.

Los resultados de la optimización fueron validados directamente en aguas de re-uso de la industria petrolera, se observó que en presencia de las fuentes nutricionales ( $\mathrm{N}$ y $\mathrm{P}$ ) hubo degradación de fenol por las cepas, a comparación con la ausencia de las fuentes. Estos nutrientes son fundamentales para el metabolismo celular debido a que incrementan la efectividad de degradación de hidrocarburos (Walworth \& Reynolds, 1995). Estos diseños estadísticos han sido utilizados en varios estudios (Annadurai et al., 2008; Agarry, Solomon, \& Layokun, 2008; Ghanem, Al-Garni \& Al-Shehri, 2009; Sridevi et al., 2011) para mejorar la degradación de fenol por medio de la optimización de nutrientes en combinación con microorganismos. El uso adecuado de estos diseños estadísticos permiten evaluar diferentes condiciones de ensayo para lograr reducir la complejidad de un problema, para este estudio, se identificaron los factores limitantes y se lograron optimizar los mismos, reduciendo el fenol en las concentraciones evaluadas en aguas de producción de hidrocarburos.

Al finalizar este trabajo se obtuvieron ocho cepas bacterianas degradadoras de fenol: cuatro cepas asociadas a biopilas (Sps1, Sps2, Sc3, Sc5) y cuatro cepas de cultivos de interés agrícola (ECO1P, ECO2P, Fenol2C y FenolP) después de enriquecimiento por 63 días en caldo de cultivo selectivo y cuatro pases sucesivos en medio solido Bushnell-Haas suplementado con fenol. La identificación por análisis del gen 16S rARN indicó que la mayoría de los aislados son Pseudomonas sp. y una cepa del género Achromobacter sp. En las cinéticas de crecimiento y degradación de fenol, la cepa Pseudomonas sp. Sps1 presentó la mejor capacidad de remoción del compuesto a $1200 \mathrm{mg}$ / L, degradándolo totalmente en 96 h. Se observó que las fases de adaptación de las cepas analizadas a las concentraciones de fenol utilizadas fueran diferentes entre sí, sugiriendo que su comportamiento de crecimiento y la velocidad de degradación están relacionados con esta fase de adaptación. Por medio de los diseños experimentales empleados fue posible identificar las fuentes nutricionales limitantes para la degradación de fenol como lo fueron el $\mathrm{N}$ y $\mathrm{P}$, y estimular la remoción de fenol en aguas de re-uso provenientes de producción de hidrocarburos. Se demostró que por medio de la utilización de microorganismos junto con la aplicación de nutrientes, se logró disminuir el fenol presente en aguas tratadas de re-uso provenientes de la industria petrolera, logrando obtener una alternativa para la disminución de este compuesto el cual puede perjudicar los sistemas de producción agrícola al utilizar esta agua como una alternativa de riego en épocas de sequía. 


\section{AGRADECIMIENTOS}

Este trabajo fue financiado por el convenio de cooperación técnica CORPOICA ECOPETROL DHS-1754, apoyado por María Angélica Pichimata desde la Coordinación de Laboratorios de CORPOICA, Andrés Moreno, investigador del Laboratorio de Microbiología de Suelos (CORPOICA) y por Anngie Hernández, Investigador Master (CORPOICA).

\section{RESUMEN}

La explotación del petróleo y el uso de sus derivados han contribuido para el desarrollo tecnológico a nivel mundial. Esta actividad, no obstante, genera muchos subproductos los cuales atentan contra la salud del ambiente. Las aguas de producción, por ejemplo, pueden contener trazas de metales pesados e hidrocarburos, razón por la cual deben ser reincorporadas de forma adecuada al ambiente. En este trabajo, se aislaron, caracterizaron e identificaron molecularmente ocho bacterias en base a su capacidad para degradar fenol. Primero, se evaluó el crecimiento y la degradación de fenol bajo diferentes concentraciones (500, 800 y $1200 \mathrm{mg} / \mathrm{L})$, y posteriormente se emplearon diseños estadísticos para la selección de condiciones óptimas de degradación. Los resultados mostraron que las ocho cepas evaluadas fueron capaces de usar el fenol como fuente única de carbono; no obstante, las cinéticas de degradación y la máxima tolerancia de las cepas al fenol variaron ampliamente. Debido a su tolerancia y capacidad para metabolizar fenol, la cepa Pseudomonas sp. Sps1 fue seleccionada para posteriores estudios. Mediante el uso de un diseño de Plackett Burman y un diseño factorial fraccionado en el cual diferentes fuentes de carbono, nitrógeno, y fósforo fueron evaluadas y degradación de fenol fue usado como variable de respuesta, fue posible seleccionar las condiciones óptimas para la degradación de fenol por Sps1. Finalmente, se emplearon estas condiciones para evaluar la degradación de fenol en aguas de re-uso provenientes directamente de la industria petrolera. Los resultados, mostraron que la bioestimulación favoreció el proceso de degradación del fenol comparado con el control. En conclusión, en este estudio se demostró que la bioaumentación con bacterias nativas acompañada de bioestimulación generan una tecnología económica y ambientalmente amigable para la remoción de contaminantes en aguas residuales tratadas de la industria petrolera.

Palabras clave: extracción de crudo, hidrocarburo, bacterias nativas, nutrientes, Colombia.

\section{REFERENCIAS}

Agarry, S. E., Solomon, B. O., \& Layokun, S. K. (2008). Optimization of process variables for the microbial degradation of phenol by Pseudomonas aeruginosa using response surface methodology. African Journal of Biotechnology, 7(14), 2409-2416.

Annadurai, G., Ling, L. Y., \& Lee, J. F. (2008). Statistical optimization of medium components and growth conditions by response surface methodology to enhance phenol degradation by Pseudomonas putida. Journal of Hazardous Materials, 151(1), 171-8.

Arthur, J., Langhus, B., \& Patel, C. (2005). Technical Summary of Oil \& Gas Produced Water Treatment Technologies. Tulsa, Oklahoma, USA, ALL CONSULTING, LLC, 1-53.

Bandyopadhyay, K., Das, D., \& Maiti, B. R. (1998). Kinetics of phenol degradation using Pseudomonas putida MTCC 1194. Bioprocess Engineering, 18(5), 373-77.

Banerjee, A., \& Ghoshal, A. K. (2010). Phenol degradation by Bacillus cereus: pathway and kinetic modeling. Bioresource Technology, 101(14), 5501-7.

Bushnell, L. D., \& Hass, H. F. (1941). The utilization of certain hydrocarbons by microorganisms. Journal of Bacteriology, 41(5), 653-73.

Caballero-Mellado, J., Onofre-Lemus, J., Estrada-de Los Santos, P., \& Martínez-Aguilar, L. (2007). The tomato rhizosphere, an environment rich in nitrogen-fixing Burkholderia species with capabilities of interest for agriculture and bioremediation. Applied and Environmental Microbiology, 73(16), 5308-19.

Contreras, E. M., Albertario, M. E., Bertola, N. C., \& Zaritzky, N. E. (2008). Modelling phenol biodegradation by activated sludges evaluated through respirometric techniques. Journal of Hazardous Materials, 158(2-3), 366-74.

Cordova-Rosa, S. M., Dams, R. I., Cordova-Rosa, E. V., Radetski, M. R., Corrêa, A. X. R., \& Radetski, C. M. (2009). Remediation of phenol-contaminated soil by a bacterial consortium and Acinetobacter calcoaceticus isolated from an industrial wastewater treatment plant. Journal of Hazardous Materials, 164(1), 61-6.

Dong, X., Hong, Q., He, L., Jiang, X., \& Li, S. (2008). Characterization of phenol-degrading bacterial strains isolated from natural soil. International Biodeterioration and Biodegradation, 62(3), 257-262.

Durell, G., Røe Utvik, T., Johnsen, S., Frost, T., \& Neff, J. (2006). Oil well produced water discharges to the North Sea. Part I: Comparison of deployed mussels (Mytilus edulis), semi-permeable membrane devices, 
and the DREAM model predictions to estimate the dispersion of polycyclic aromatic hydrocarbons. Marine Environmental Research, 62(3), 194-223.

Fakhru'l-Razi, A., F. R., Pendashteh, A., Abdullah, L. C., Biak, D. R., Madaeni, S. S., \& Abidin Z. Z. (2009). Review of technologies for oil and gas produced water treatment. Journal of Hazardous Materials, $170(2-3), 530-51$.

Geng, A., Soh, A. E. W., Lim, C. J., \& Loke, L. C. T (2006). Isolation and characterization of a phenoldegrading bacterium from an industrial activated sludge. Applied Microbiology and Biotechnology, 71(5), 728-35.

Ghanem, K. M., Al-Garni, S. M., \& Al-Shehri A. N. (2009). Statistical optimization of cultural conditions by response surface methodology for phenol degradation by a novel Aspergillus flavus isolate. African Journal of Biotechnology, 8(15), 3576-3583.

Ho, Y. N., Mathew, D. C., Hsiao, S. C., Shih, C. H., Chien, M. F., Chiang, H. M., \& Huang, C. C. (2012). Selection and application of endophytic bacterium Achromobacter xylosoxidans strain F3B for improving phytoremediation of phenolic pollutants. Journal of Hazardous Materials, 219(220), 43-49.

Jain, R. K., Kapur, M., Labana, S., Lal, B., Sarma, P. M., Bhattacharya, D., \& Thakur, I. S. (2005). Microbial diversity: application of microorganisms for the biodegradation of xenobiotics. Current Science, $89(1), 10$

Jung, W. K., \& Neal, E. A. I. (1991). A comprehensive study on the biological treat abilities of phenol and methanol. II. The effects of temperature, $\mathrm{pH}$, salinity and nutrients. Water Research, 15, 1233-1247.

Kotresha, D., \& Vidyasagar, G. M. (2007). Isolation and characterisation of phenol-degrading Pseudomonas aeruginosa MTCC 4996. World Journal of Microbiology and Biotechnology, 24, 541-7.

Lin, J., Reddy, M., Moorthi, V., \& Qoma, B. E. (2008). Bacterial removal of toxic phenols from an industrial effluent. African Journal of Biotechnology, $7(13), 2232-8$

Masque, C., Nolla, M., Bordons, A., Tarragona, D., \& Barcelona, U. D. (1987). Selection and adaptation of a phenol-degrading strain of Pseudomonas. Biotechnology Letters, 9(9), 655-60.

Michałowicz, J., \& Duda, W. (2007). Phenols - sources and toxicity. Polish Journal of Environmental Studies, 16(3), 347-62.
Mohite, B. V., Jalgaonwala, R. E., Pawar, S., \& Morankar, A. (2010). Isolation and characterization of phenol degrading bacteria from oil contaminated soil. Innovative Romanian Food Biotechnology, 7, 61-5.

Nair, C. I., Jayachandran, K., \& Shashidhar, S. (2008). Biodegradation of phenol. African Journal of Biotechnology, 7(25), 4951-4958.

Naresh, B., Honey, P., \& Vaishali, S. (2012). Biodegradation of phenol by a bacterial strain isolated from a phenol contaminated site in India. Research Journal of Environment Sciences, 1(1), 46-9.

Quan, X., Shi, H., Liu, H., Wang, J., \& Qian, Y. (2004). Removal of 2,4-dichlorophenol in a conventional activated sludge system through bioaugmentation. Process Biochemistry, 39(11), 1701-7.

Ren, J., Lin, W. T., Shen, Y. J., Wang, J. F., Luo, X. C., \& Xie, M. Q. (2008). Optimization of fermentation media for nitrite oxidizing bacteria using sequential statistical design. Bioresource Technology, 99(17), 7923-7.

Sikkema, J., de Bont, J. A., \& Poolman, B. (1995). Mechanisms of membrane toxicity of hydrocarbons. Microbiological Reviews, 59(2), 201-22.

Singleton, V. L., \& Rossi, J. A. Jr. (1965). Colorimetry of total phenolics with phosphomolybdic-phosphotungstic acid reagents. American Journal of Enology and Viticulture, 16, 144-158.

Sridevi, V., Lakshmi, M. V. V. C., \& Swamy, A. V. N. (2011). Implementation of response surface methodology for phenol degradation using Pseudomonas putida (NCIM 2102). Journal of Bioremediation \& Biodegradation, 02(02).

Stanbury, P. F., Whitaker, A., \& Hall, S. J. (1986). Media for industrial fermentations. In: Principles of Fermentation Technology (93-122). Oxford: Pergamon Press.

Walworth, J. L., \& Reynolds, C. M. (1995). Bioremediation of a petroleum-contaminated cryic soil: effects of phosphorus, nitrogen, and temperature. Journal of Soil Contamination, 4(3), 299-310.

Yang, C., \& Lee, C. (2007). Enrichment, isolation, and characterization of phenol-degrading Pseudomonas resinovorans strain $\mathrm{P}-1$ and Brevibacillus sp. strain P-6. International Biodeterioration and Biodegradation, 59(3), 206-10. 
